\title{
Comment on "Study of Systematic Bias in Measuring Surface Deformation With SAR Interferometry" by Ansari et al. (2021)
}

\author{
Claudio De Luca, Francesco Casu, Michele Manunta, Giovanni Onorato and Riccardo Lanari, Fellow, \\ IEEE
}

\begin{abstract}
In a recent publication Ansari et al. (2021) [1] claim (see, in particular, the Discussion and Recommendation Section in their article) that the advanced differential SAR interferometry (InSAR) algorithms for surface deformation retrieval, based on the small baseline approach, are affected by systematic biases in the generated InSAR products. Therefore, to avoid such biases, they recommend a strategy primarily focused on excluding "the short temporal baseline interferograms and using long baselines to decrease the overall phase errors". In particular, among various techniques, Ansari et al. (2021) [1] identify the solution presented by Manunta et al. (2019) [2] as a small baseline advanced InSAR processing approach where the presence of the above-mentioned biases (referred to as a fading signal) compromises the accuracy of the retrieved InSAR deformation products.
\end{abstract}

We show that the claim of Ansari et al. (2021) [1] is not correct (at least) for what concerns the mentioned approach discussed by Manunta et al. (2019) [2]. In particular, by processing the Sentinel-1 dataset relevant to the same area in Sicily (southern Italy) investigated by Ansari et al. (2021) [1], we demonstrate that the generated InSAR products do not show any significant bias.

Index Terms- P-SBAS, interferometric synthetic aperture radar (SAR) (InSAR), multilook interferograms, distributed scatterers (DSs), systematic bias, phase inconsistencies, phase unwrapping errors, time series analysis.

\section{INTRODUCTION}

$\mathrm{T}$ HE "non-closure behavior" of the multi-look interferometric SAR (InSAR) phase signals relevant to distributed scatterers (DS) represents the starting point of the analysis presented by Ansari et al. (2021) [1] (see formula 1 and 2 of the article in question). This is a rather well known concept for the InSAR community that in the last twenty years have been using temporal sequences of multi-look interferograms for the retrieval of surface deformation time series and the corresponding mean velocity maps. In particular, for what concerns the original Small BAseline Subsets (SBAS) approach [3], this issue has been the main clue for the subsequent exploitation of triangulation networks in the temporal-perpendicular baseline plane to select the small baseline interferometric data pairs to be used for the generation of multi-temporal sequences of multi-look interferograms and the subsequent phase unwrapping step of these data [4-8]. We remark that this triangulation-based ${ }^{1}$ strategy is the key point for the evaluation of the "triangular coherence", the parameter used to select the pixels to be considered in the phase unwrapping procedure and in the subsequent steps of the SBAS processing chain, as clearly and extensively described in [2],[4],[5].

Moreover, following the first developments of the original SBAS approach, the information relevant to the non-closure of the phase differences triplets has been further exploited in order to retrieve the quasi-irrotational multi-temporal, multilook InSAR phase signals relevant to the pixels characterized by high triangular coherence values. This is, in particular, the case of the algorithm described in [9], which implements a nonlinear minimization problem based on retrieving, from a redundant set of multi-look interferograms, the wrapped phase component that, for each pixel, minimizes the weighted circular variance of the phase difference between the original and the retrieved multi-look interferograms.

It is also worth noting that, when dealing with the so-called first generation space-borne SAR sensors (i.e., ERS, ENVISAT, RADARSAT), characterized by (nearly) monthly revisit time and an orbital tube diameter significantly greater than $1 \mathrm{~km}$, the main source of the non-closure of the multilook phase difference triplets is clearly represented by the decorrelation noise [10]. On the other hand, nowadays, particularly in the case of the Sentinel-1 (S-1) constellation, characterized by small orbital tube diameter (less than 300 meters) and short revisit time (down to 6 days, in the best case), new effects can play a significant role in the multi-look interferometric phase, as described by Ansari et al. (2021) [1]. However, for the pixels characterized by high triangular coherence values, solutions like the one described in [9] are capable, in few iterations, to retrieve the quasi-irrotational multi-look InSAR phase components and, therefore, to prevent the presence of artifacts (including a possible bias, although we typically refer to noise [2]) in the deformation time series. Accordingly, we present in the following a dedicated experiment demonstrating that the claim of Ansari et al. (2021) [1] is not correct (at least) for the approach proposed by Manunta et al. (2019) [2] because no significant bias affects the deformation time series retrieved with such a small baseline InSAR processing solution. 


\section{A. Sentinel-1 dataset}

The InSAR analysis presented in this Comment involves a S-1 dataset acquired from ascending orbits (track 44) over Sicily (Southern Italy), representing the same area and the same acquisition geometry of the main test site investigated by Ansari et al. (2021) [1], whose main characteristics are summarized in Table I. We also remark that the dataset processed in this Comment extends from May 2016 to May 2020 and slightly differs from that used in [1], which is instead relevant to the October 2014 - September 2018 time interval. Our choice is justified by an uncontrolled change in the S-1 processor that makes the Instrument Processing Facility (IPF) versions from the 2.60 to 2.70 releases (corresponding to the time interval from November 2015 to April 2016) incompatible, from the interferometric point of view, with different IPF versions (more details can be found in
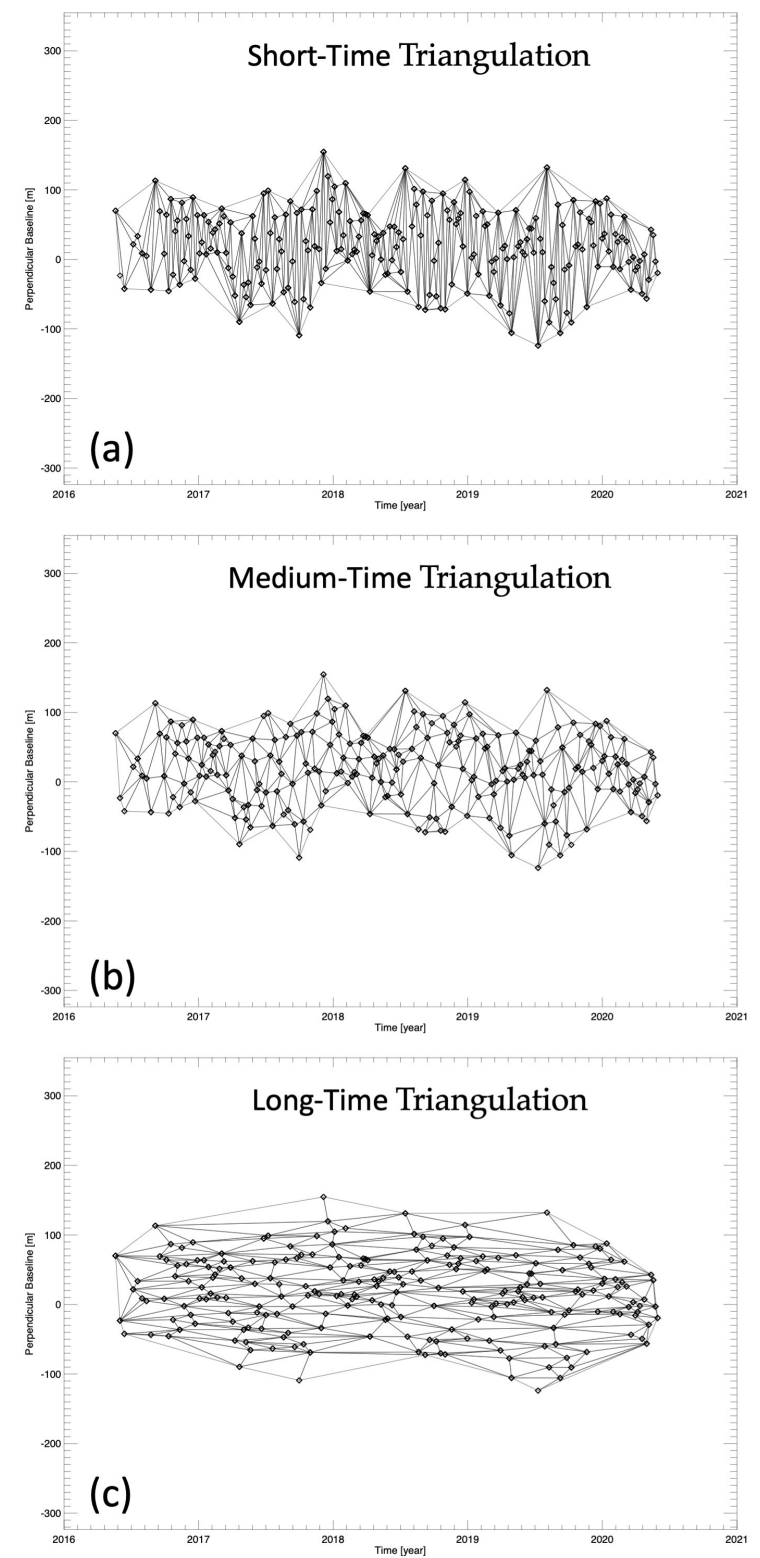

Fig. 1. Reduced Delaunay triangulations representing the three interferograms networks exploited in the presented experiment: (a) shorttime triangulation; (b) medium-time triangulation; (c) long-time triangulation.
[11]). Because of this issue, which may introduce significant artifacts in the generated interferograms (see [2]), we decide to reject all the S-1 data acquired before May 2016.

TABLE I

MAIN CHARACTERISTICS OF THE EXPLOITED SENTINEL-1 SAR DATASET

\begin{tabular}{l|c}
\hline \hline Acquisition geometry & Ascending orbits \\
\hline Wavelength & $5.5 \mathrm{~cm}$ \\
\hline Acquisition mode & TOPS \\
\hline Mean look-angle & $\sim 39^{\circ}$ \\
\hline Pixel spacing & $\sim 80 \mathrm{~m} \mathrm{x} 80 \mathrm{~m}$ \\
\hline Track & 44 \\
\hline Time interval & $18 / 05 / 2016-27 / 05 / 2020$ \\
\hline Number of SAR acquisitions & 230 \\
\hline \hline
\end{tabular}

However, in order to be as much as possible consistent with the dataset used by Ansari et al. (2021) [1], we preserve the 4year time interval by including all the S-1 images acquired, over the area of interest, until May 2020. It is worth noting that the investigated time period includes the volcano-tectonic crisis of the Mount Etna occurred in December 2018 [12], whose deformation signals are relevant for some of the following considerations.

\section{B. Rationale of the presented experiment}

The experiment presented in the following section is based on the exploitation of the S-1 P-SBAS processing chain described in [2] that also includes, as already underlined, the processing procedure discussed in [9]. In particular, once the quasi-irrotational multi-look interferometric phases are retrieved, we select for our experiment the interferogram sequences relevant to three reduced Delaunay triangulations computed in the temporal-perpendicular baseline plane. Such triangulations, that we label as "short-time", "medium-time" and "long-time", have a different distribution of the temporal baselines, as clearly shown in Fig. 1 (a), (b), and (c), respectively.

Each interferometric dataset relevant to the three triangulations is then unwrapped and inverted by means of the method presented in [2], starting from the pixels characterized by high values of the triangular coherence, defined as follows $[2,5]$ :

$$
\Gamma_{t r}(P)=\frac{1}{\Lambda}\left|\sum_{1}^{\Lambda} \exp [j C \cdot \varphi(P)]\right| \in[0,1]
$$

wherein $\Lambda$ is the number of triangles of the exploited triangulation, $C$ is the triangulation matrix and $\varphi(P)$ is the vector of the interferometric phases of the pixel $P$.

The achieved deformation time series and the corresponding velocity maps are finally compared each other. Clearly, if any bias related to the different temporal baselines values of the interferometric sequences is present in the deformation time series, such a comparison must certainly point out it. The following section is focused on the results of such a comparison. 

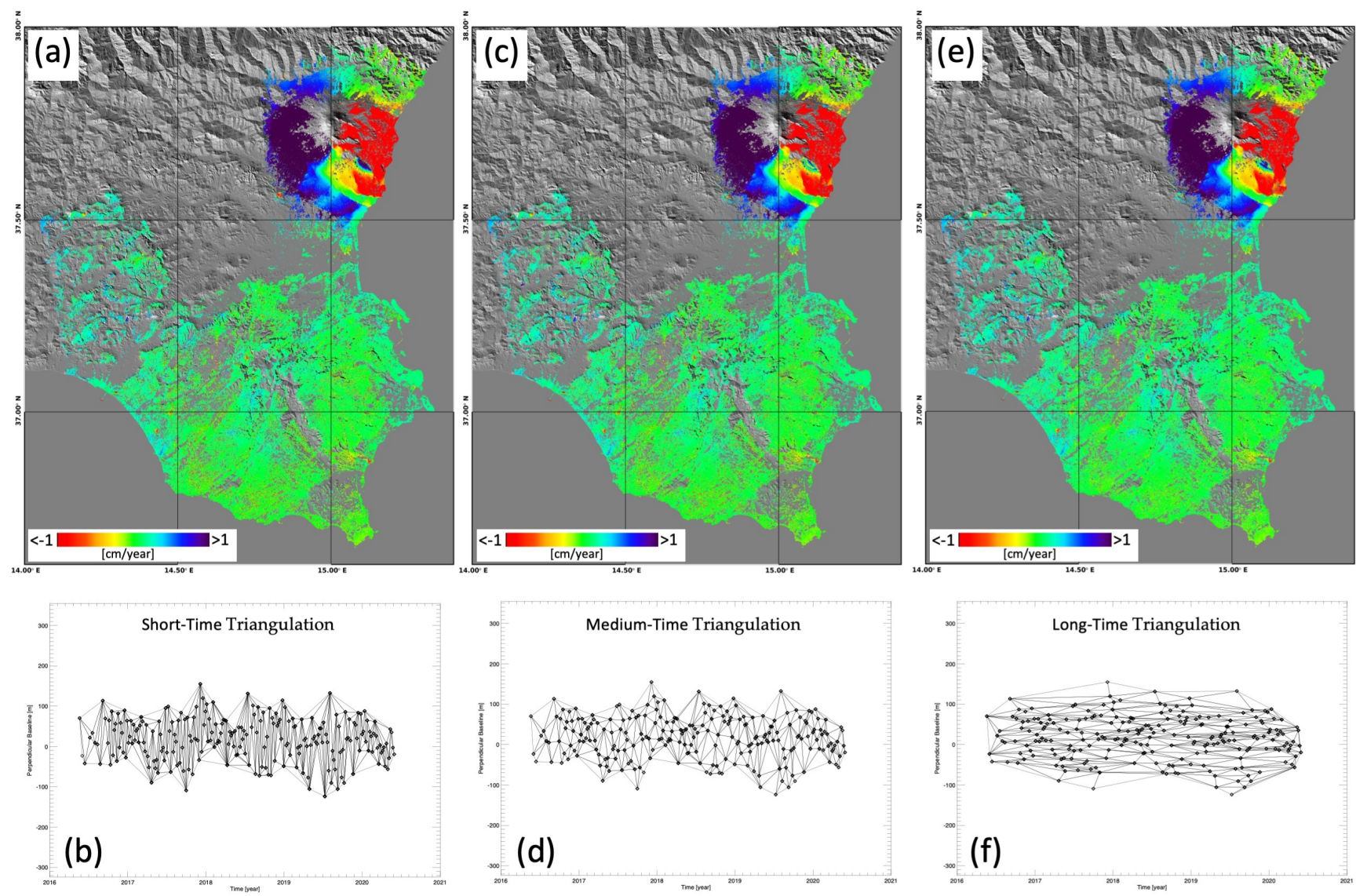

Fig. 2. Mean deformation velocity maps retrieved through the P-SBAS processing chain, by exploiting the three different interferograms networks relevant to the triangulations shown in Fig. 1: (a) is the mean velocity map generated by considering the short-time triangulation represented in (b); (c) is the mean velocity map generated by considering the medium-time triangulation represented in (d); (e) is the mean velocity map generated by considering the longtime triangulation represented in (f). Note that the interferogram networks shown in panel (b), (d) and (f) are the same of panels (a), (b) and (c) of Figure 1, respectively, but they are repeated here for sake of completeness.

\section{RESULTS}

We present in Fig. 2 the mean deformation velocity maps estimated from the deformation time series obtained by applying the P-SBAS approach described in [2] to the multilook interferogram sequences relevant to the three interferometric networks (triangulations) described in the previous section (see Fig. 1). We again remark that the presented results are relevant to the same area analyzed in [1], although it is not fully clear the reason why that analysis was limited to this zone only, whereas the S-1 frame is significantly wider. However, the readers may freely access the three wrapped interferograms sequences relevant to the S1 full-frame, provided as supplementary material to this Comment [13], in order to carry out their own analysis in the overall area.

Fig. 2 (a) and (b) show the mean deformation velocity map and the relevant interferometric network for the "short-time" triangulation case, while Fig. 2 (c), (d), and (e), (f) show the corresponding information for the "medium-time" and "longtime" triangulations, respectively. Note that for a fully consistent comparison of the achieved results, the three velocity maps include only those pixels coherent in all the three interferometric analyses (short-, medium-, and longtime). In particular, we consider as coherent the pixels with values greater than a selected threshold (0.95) of the temporal coherence defined as follows $[4,5]$ :

$$
\begin{aligned}
\Gamma_{t m p}(P)=\frac{1}{\mathrm{M}} \mid \sum_{1}^{\mathrm{M}} \exp [ & j(\varphi(P)-\hat{\varphi}(P))] \mid \\
= & \frac{1}{\mathrm{M}}\left|\sum_{1}^{\mathrm{M}} \exp \left[j\left(\varphi(P)-A \cdot \hat{\varphi}_{T S}(P)\right)\right]\right| \in[0,1]
\end{aligned}
$$

wherein $\mathrm{M}$ is the number of the interferograms of the exploited triangulation, $A$ is the incidence matrix of the oriented graph associated with the interferometric pairs, $\hat{\varphi}(P)$ is the vector of the unwrapped interferometric phases "regenerated" from the retrieved time series vector, say $\hat{\varphi}_{T S}(P)$.

It is quite evident, by inspecting Fig. 2, that the obtained results do not show any significant bias. However, to indisputably confirm this claim, we compute in Fig. 3 the differences between the three deformation velocity maps of Fig. 2. Fig. 3 (a) shows the difference between the short- and medium-time velocity maps, whereas Fig. 3(b) is relevant to the short- and long-time comparison and Fig. 3(c) to mediumand long-time one. It is evident, once again, the total absence of any bias or fading signal. For the sake of completeness, in Fig. 3 we also report some time series differences (see plots of the point P1 and P2) selected in areas where, according to the experiments shown in Ansari et al. (2021) [1], the pixels should be strongly affected by a systematic bias, while it is evident that in our results there is no bias at all. 

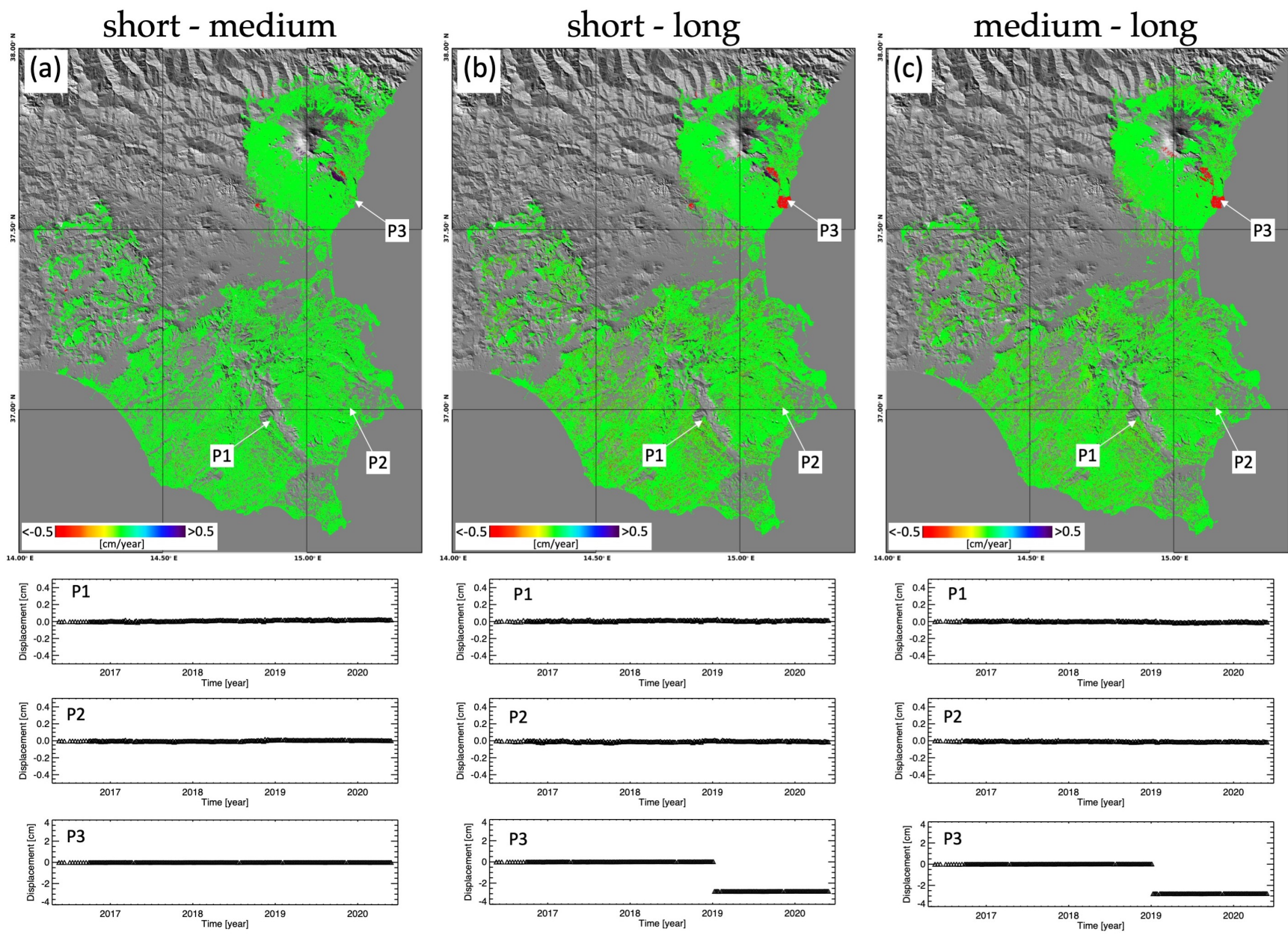

Fig. 3. Differences among the P-SBAS results retrieved with the three reduce Delaunay triangulations. (a) is the difference between the mean deformation velocity maps of short- and medium-time triangulation. (b) is the difference between the mean deformation velocity maps of short- and long-time triangulation. (c) is the difference between the mean deformation velocity maps of medium- and long-time triangulation. The plots shown the deformation time series differences for some relevant pixels of the area, identified by P1, P2 and P3 labels

The unique differences we can observe in Fig. 3 (a), (b), and (c) are spatially very localized and clearly due to phase unwrapping errors, as highlighted by the deformation time series differences (see P3 in Fig. 3). In particular, such time series point out that the discrepancies take temporally place in December 2018, when the volcano-tectonic crisis of the Mount Etna occurred, and are spatially limited to the areas affected by the induced ground deformations [12]. Note also that, in this case the main discrepancies appear when considering the longer baseline interferograms (see the plots related to the pixel P3 in Fig. 3(b) and (c)), which are typically less immune to the phase unwrapping errors, representing the main causes of the only inconsistencies we detect.

\section{DISCUSSION AND RECOMMENDATION}

It is evident from the results presented in the previous section that the claim of Ansari et al. (2021) [1], about the presence of phase artefacts (referred to as biases or fading signal) due to short temporal baseline interferograms, is incorrect (at least) if we consider the S-1 P-SBAS processing chain presented in [2]. Indeed, we show that this InSAR approach permits to guarantee fully consistent solutions when dealing with multi-look interferograms sequences characterized by short-, medium- and long-time temporal baselines, as long as the interferograms sequences are properly generated and processed.

We also note that some areas of the presented results are affected by localized inconsistencies but they are due to phase unwrapping errors taking temporally place in correspondence of the December 2018 volcano-tectonic crisis of the Mount Etna. These errors are clearly (but not surprisingly) affecting the longer temporal baseline interferograms.

Moreover, our findings also allow us to confirm that the short temporal baseline interferograms permit to guarantee a wide area coverage thanks to the large number of pixels with high temporal coherence values. In particular, to clearly show the benefit of using small (temporal) baseline interferograms, in terms of pixels density, we present in Fig. 4 the coherent pixels maps (temporal coherence greater than 0.95) for the short- and long-time triangulation analyses (see Fig 4 (a) and (b), respectively). By comparing Fig. 4 (a) and (b) the achieved improvement is evident. Quantitatively, we pass from 1.811.581 coherent pixels of the short-time triangulation (Fig. 4 (a)) to 1.127 .922 pixels of the long-time one (Fig. 4 (b)), with a decrease of about $40 \%$. These results confirm once again the relevance of properly exploiting short temporal baseline interferograms in advanced multi-temporal InSAR approaches. 

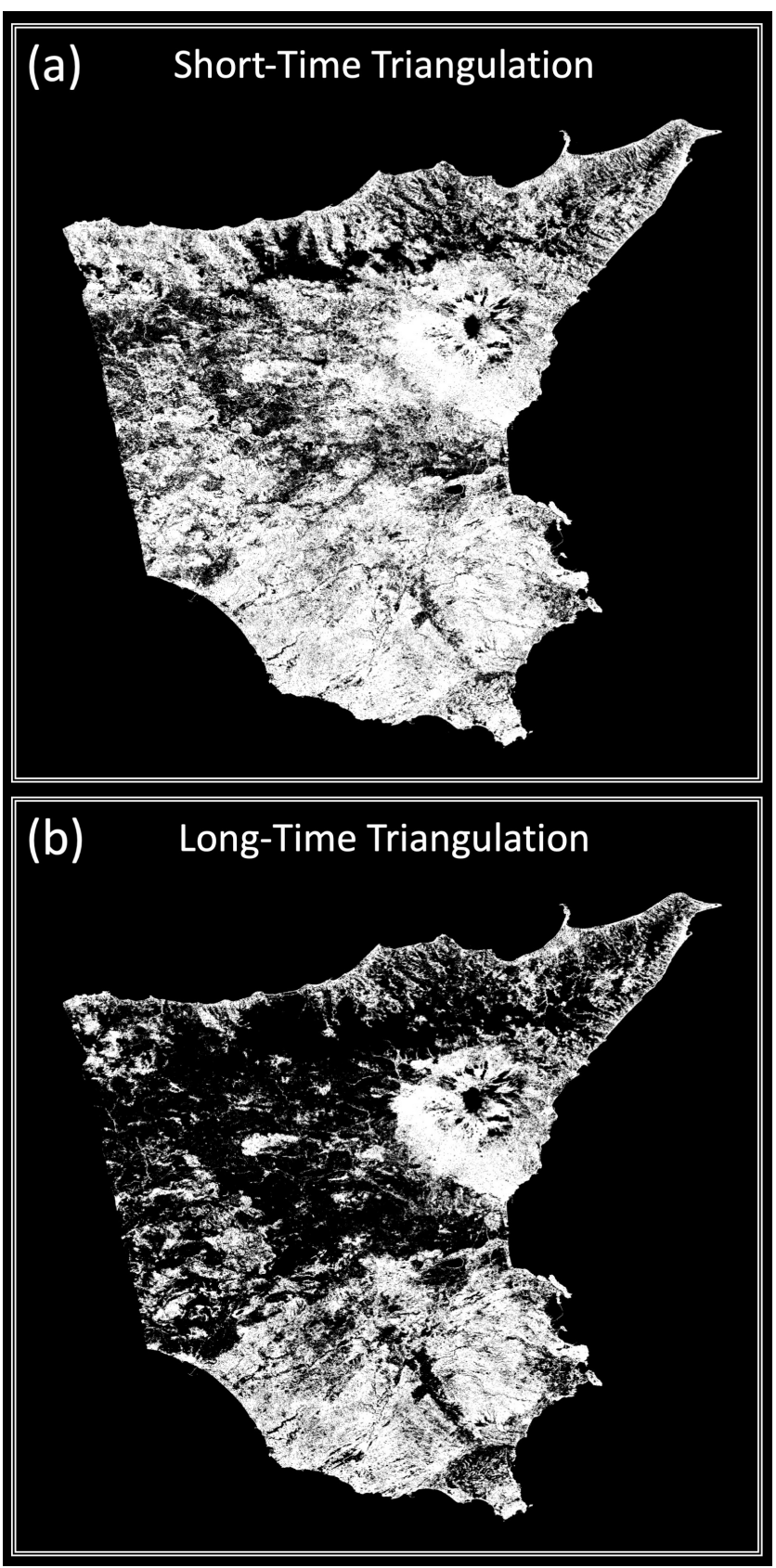

Fig. 4. Map of the pixels showing a temporal coherence (computed according to (2)) greater than 0.95 in dependence of the exploited (a) short-time (see Fig. 1(a)) and (b) long-time (see Fig. 1(c)) triangulations.

\section{CONCLUSION}

We have shown in this Comment that, differently from what claimed by Ansari et al. (2021) [1], the advanced InSAR processing approach presented by Manunta et al. (2019) [2], based on the exploitation of small baseline interferograms and including the processing stage discussed in [9], can be effectively applied to generate InSAR products that do not present any significant bias. In particular, we have disproved the claim of Ansari et al. (2021) [1] by processing, through the P-SBAS approach [2], three multi-look interferogram sequences characterized by different temporal baselines, relevant to the Sentinel-1 data of the Sicily area (southern
Italy) investigated by Ansari et al. (2021) [1]. Specifically, we show that the achieved results are fully consistent for the three interferograms sequences, thus proving that the accuracy of the retrieved InSAR deformation products is preserved. Moreover, the presented results further confirm the valuable contribution of small baseline advanced processing schemes that, if properly implemented, permit to mitigate the impact of phase unwrapping errors and to significantly improve the spatial coverage characterizing the achieved InSAR results.

\section{ACKNOWLEDGMENT}

This research was funded by the "Presidenza del Consiglio dei Ministri-Dipartimento della Protezione Civile" (Presidency of the Council of Ministers-Department of Civil Protection), through the IREA-DPC agreements, and the EPOS-SP projects of the European Union Horizon 2020 for Research and Innovation Program under Grant 871121.

\section{REFERENCES}

[1] H. Ansari, F. De Zan and A. Parizzi, "Study of Systematic Bias in Measuring Surface Deformation With SAR Interferometry," in IEEE Transactions on Geoscience and Remote Sensing, vol. 59, no. 2, pp. 1285-1301, Feb. 2021, doi: 10.1109/TGRS.2020.3003421.

[2] Manunta, M.; De Luca, C.; Zinno, I.; Casu, F.; Manzo, M.; Bonano, M.; Fusco, A.; Pepe, A.; Onorato, G.; Berardino, P.; De Martino, P.; Lanari, R. The Parallel SBAS Approach for Sentinel-1 Interferometric Wide Swath Deformation Time-Series Generation: Algorithm Description and Products Quality Assessment, IEEE Trans. Geosci. Remote Sens., 2019, 57

[3] P. Berardino, G. Fornaro, R. Lanari, and E. Sansosti, "A new algorithm for surface deformation monitoring based on small baseline differential SAR interferograms," IEEE Trans. Geosci. Remote Sens., vol. 40, no. 11, pp. 2375-2383, Nov. 2002.

[4] A. Pepe and R. Lanari, "On the Extension of the Minimum Cost Flow Algorithm for Phase Unwrapping of Multitemporal Differential SAR Interferograms," in IEEE Transactions on Geoscience and Remote Sensing, vol. 44, no. 9, pp. 2374-2383, Sept. 2006, doi: 10.1109/TGRS.2006.873207.

[5] A. Pepe, Advanced Differential Interferometric SAR Techniques, the Extended Minimum Cost Flow Phase Unwrapping (EMCF) Technique. Saarbrucken, Germany: VDM Verlag, 2009.

[6] A. Pepe, L. D. Euillades, M. Manunta and R. Lanari, "New Advances of the Extended Minimum Cost Flow Phase Unwrapping Algorithm for SBAS-DInSAR Analysis at Full Spatial Resolution," in IEEE Transactions on Geoscience and Remote Sensing, vol. 49, no. 10, pp. 4062-4079, Oct. 2011, doi: 10.1109/TGRS.2011.2135371.

[7] A. Pepe, P. Berardino, M. Bonano, L. D. Euillades, R. Lanari and E. Sansosti, "SBAS-Based Satellite Orbit Correction for the Generation of DInSAR Time-Series: Application to RADARSAT-1 Data," in IEEE Transactions on Geoscience and Remote Sensing, vol. 49, no. 12, pp. 5150-5165, Dec. 2011, doi: 10.1109/TGRS.2011.2155069.

[8] A. Pepe, A. Bertran Ortiz, P. R. Lundgren, P. A. Rosen and R. Lanari, "The Stripmap-ScanSAR SBAS Approach to Fill Gaps in Stripmap Deformation Time Series With ScanSAR Data," in IEEE Transactions on Geoscience and Remote Sensing, vol. 49, no. 12, pp. 4788-4804, Dec. 2011, doi: 10.1109/TGRS.2011.2167979.

[9] A. Pepe, Y. Yang, M. Manzo, and R. Lanari, "Improved EMCF-SBAS processing chain based on advanced techniques for the noise-filtering and selection of small baseline multi-look DInSAR interferograms," IEEE Trans. Geosci. Remote Sens., vol. 53, no. 8, pp. 4394-4417, Aug. 2015.

[10] H. A. Zebker and J. Villasenor, "Decorrelation in interferometric radar echoes," IEEE Trans. Geosci. Remote Sens., vol. 30, no. 5, pp. 950959, Sep. 1992. 
[11] https://qc.sentinel1.groupcls.com/product/S1/MET DISCLM/000058/ S1A OPER MET DISCLM MPC 20210128T101840 I000058/S1 QD 58.pdf

[12] De Novellis, V., Atzori, S., De Luca, C., Manzo, M., Valerio, E., Bonano, M., et al. (2019). DInSAR analysis and analytical modeling of Mount Etna displacements: The December 2018 volcanotectonic crisis. Geophysical Research Letters, 46, 5817- 5827.

[13] Michele Manunta, Claudio De Luca, Francesco Casu, Giovanni Onorato, Riccardo Lanari, May 27, 2021, "Sentinel-1 P-SBAS multilook interferogram sequences and triangulation networks", IEEE Dataport, doi: https://dx.doi.org/10.21227/f0tq-4b68. 